\title{
Formación de secretarios en la práctica de valores éticos y morales en la atención al público
}

\section{Training of secretaries in the practice of ethical and moral values in customer service}

\section{Formación secretarios en la práctica de valores éticos}

\author{
Reynier García Rodríguez \\ Instituto Superior Tecnológico Portoviejo. Manabí, Ecuador. email: reygarod79@gmail.com ORCID: \\ https://orcid.org/0000-0002-4160-5749
}

Recibido: 18-02-2020 Aprobado: 06-04-2020

\begin{abstract}
Resumen
El estudio realizado es parte de un proyecto de investigación que ejecutó en el año 2019 un grupo de docentes de la Carrera de Secretariado, con el financiamiento del Instituto Superior Tecnológico Portoviejo (ITSUP). El objetivo fue determinar la repercusión de la formación académica de secretarios en la práctica de valores éticos y morales en la atención al público. La investigación es de tipo descriptiva de cohorte transversal, los métodos utilizados fueron el histórico lógico y el análisissíntesis. En el estudio participaron 200 secretarios de empresas públicas y privadas de la ciudad de Portoviejo, en Ecuador. Se aplicó un cuestionario dirigido a la práctica de valores éticos y morales en la atención al público, obteniendo como resultado que es reducido el porcentaje de usuarios que se encontró "muy satisfecho" por la atención recibida, acentuándose la mayor de las respuestas en el ítem de "satisfechos", destacando como conclusión lo importante que es la formación ética de las secretarios para un elevado nivel de calidad en la atención al público.
\end{abstract}

Palabras Clave: atención al cliente, axiología, talento humano

\footnotetext{
Abstract

The study carried out is part of a research project carried out in 2019 by a group of teachers in the Secretarial Career, with funding from the Portoviejo Higher Technological Institute (ITSUP). The objective was to determine the impact of the academic training of secretaries on the practice of ethical and moral values in customer service. The
}

research is descriptive type of cross-sectional cohort, the methods used were the historical $\log$ and the analysis-synthesis. 200 secretaries of public and private companies from the city of Portoviejo, in Ecuador, participated in the study. A questionnaire aimed at the practice of ethical and moral values in customer service was applied, obtaining as a result that the percentage of users who were "very satisfied" with the care received was reduced, accentuating the greater number of responses in the item of "satisfied", highlighting in conclusion how important the ethical training of secretaries is for a high level of quality in customer service.

Keywords: customer service, axiology, human talent

\section{Introducción}

La educación ética y de valores abarca una amplia variedad de aspectos, marcos conceptuales, temas y enfoques. Surgiendo del campo de la ética, ante todo tiene que ser sensible a una naturaleza antropológica multidimensional y profunda del ser humano y al reconocimiento de esto en los procesos educativos. La naturaleza relacional y comunitaria de la ética (que surge del reconocimiento de un ser humano como ser relacional, un ser de comunidad y un ser de diálogo) es extremadamente importante y dicta reflexiones sobre la justicia, la solidaridad, la compasión y la cooperación en el espíritu de un diálogo genuino en el campo de la ética y la educación en valores, que exigen aún más apertura, reciprocidad y reconocimiento mutuo. Estos aspectos son de importancia clave para la ética y la educación en valores, dado que uno de sus principales objetivos es fortalecer esa posición 
dialógica y enfática en todos los niveles del proceso educativo (Ahrne \& Brunsson, 2010).

La ética corresponde al estudio sistemático de las normas del comportamiento humano. Es la disciplina que estudia la moralidad de la conducta humana y el principio del comportamiento moral. Se relaciona con el carácter y la conducta en términos de la distinción entre saber lo que está bien de las cosas que están mal. Igualmente, la ética contribuye con el principio por el cual las personas pueden vivir juntas con éxito en una sociedad sostenible. Varios autores mencionan que la ética son los valores normativos compartidos que cualquier sociedad atesora y que se utilizan para juzgar el comportamiento o el desempeño de cualquier miembro de esa sociedad, además la establecen como el comportamiento mínimo aceptable que cualquier miembro debe lograr para ser considerado como un buen embajador de la sociedad (Alford, 2001).

La ética es más difícil de hacer cumplir que la ley como en otras profesiones, también es mejor permitir que la conciencia de los profesionales individuales sea su guía y juez. La ética se refiere a los principios fundamentales de la moralidad donde algunas acciones se etiquetan como buenas o malas, correctas o incorrectas, éticas o poco éticas. Los historiadores morales opinan que, aunque lo que se considera correcto o buen concepto de lo correcto o incorrecto, generalmente es universal. El concepto moral cambia a medida que cambia la vida social (Allen, 2002).

Una profesión es un llamado, una vacación, especialmente en una ocupación que implica educación superior o calificación técnica para ser competente y concienzudo. Es en lo que uno muestra una gran habilidad y competencia. La profesión conlleva veracidad, objetividad, honestidad y gustos, es decir, los cánones y los códigos de la profesión deben ser estrictamente tomados en cuenta, en otros para no dañar la imagen de la profesión y la secretaría como el cable vital de una empresa / organización.

Una secretaria puede ser descrita como cualquier persona asignada para mediar cuestiones de una reunión, independientemente de sus habilidades de secretaria. El Oxford Advance Learners Dictionary of Current English describe a una secretaria como un empleado en una oficina que se ocupa de la correspondencia, mantiene registros, arreglos y citas para los miembros del personal. "El catalizador", una revista de servicios públicos del estado de Oyo, describió a una secretaria como "alguien que actúa en nombre del jefe" (Ahrne \& Brunsson,
2010). Pueden considerarse como los coordinadores de trabajo administrativo de un departamento determinado. El secretario se ocupa de las actividades cotidianas del establecimiento, sirve como creador de imágenes del establecimiento para el mundo exterior y parte de esta ética es mantener en secreto toda la información en la oficina (Arce \& Macías, 2015).

La profesión de secretaria es dinámica. Esto no se debe a que necesitan secretarios en cada organización, sino a que uno de los criterios para juzgar el crecimiento y el estándar de una organización es la calidad de su servicio de secretaría. La profesión de secretaria requiere algunas cualidades, tales como: Sinceridad; una secretaria debe ser sincera en cualquier situación en la que se encuentre. Urgencia; no se espera que un secretario sea sincero en la expresión de ninguna opinión, sino que también esté activo todo el tiempo (Artico, 2013). Ella no debe mostrarse en todo su trabajo. Discreción y lealtad; la secretaria privada con frecuencia en la causa de sus deberes requerirá mucha sabiduría para manejar algunos asuntos, algunos de los cuales pueden ser de especial interés para los extraños. Sociabilidad; una secretaria no debe ser de naturaleza introvertida; es decir, debe ser del tipo que se mezcla libremente con personas tanto dentro como fuera de la empresa u organización. Sentido común e iniciativa; una secretaria necesita ejercer sentido común al manejar situaciones inesperadas, así como asuntos de rutina ordinarios (Bermúdez \& Chila, 2015).El secretario profesional ha sufrido muchos años de retroceso. Algunas de las causas del retroceso se deben a algunas actitudes poco éticas de los secretarios con respecto a su trabajo, su jefe, compañeros de trabajo y miembros del público.

La asociación de secretarios ha establecido una calificación académica mínima requerida para ingresar a la profesión. Estos incluyen: Una secretaria debe poseer una buena educación general, competencia en taquigrafía y mecanografía, buen conocimiento práctico de la cuenta y conocimiento de los procedimientos de secretaría. Deben tener interés en los asuntos actuales (Brown, Gabriel, \& Gherardi, 2009).

El objetivo del presente trabajo fue, determinar la repercusión de la formación académica de secretarios en la práctica de valores éticos y morales en la atención al público.

\section{Materiales y métodos}


La investigación es de tipo descriptiva de corte longitudinal, de tipo descriptivo, los métodos utilizados fueron el histórico lógico y el análisissíntesis. Las investigadas fueron 200 secretarios de empresas públicas y privadas de la ciudad de Portoviejo, en Ecuador durante el periodo de mayo a diciembre de 2019. La profesión de todas las participantes es variada, solamente el $18 \%$ tienen título de secretarios. Las edades de las encuestadas oscilan entre los 22 y 60 años.

Los encuestados fueron los usuarios del servicio de los secretarios. La información fue recopilada mediante un cuestionario que incluyó preguntas que miden la percepción de los usuarios frente a las secretarias sobre la actitud de éstas en la atención al público. Para contestar los cuestionarios se envió a un equipo de estudiantes que visitaran las empresas y personalmente hicieran las encuestas.

Se invitó a participar en esta investigación de forma voluntaria, se garantizó anonimato y confidencialidad a los participantes.

Se obtuvieron 205 cuestionarios contestados, pero se eliminaron cinco por estar incompletos, lo que dio un total de 200 encuestas útiles para el trabajo.

\section{Resultados}

En lo concerniente a la parte actitudinal, que responde a la práctica de valores éticos y morales de los secretarios, se presentan los resultados de 6 item que permiten hacer una valoración generalizada del comportamiento de los secretarios en la atención al público, se observa en la tabla 1.

\begin{tabular}{|c|c|c|}
\hline Opciones & Frecuencia & Porcentaje \\
\hline Muy satisfecho & 43 & $21.5 \%$ \\
\hline Satisfecho & 75 & $37.5 \%$ \\
\hline Algo satisfecho & 36 & $18.0 \%$ \\
\hline $\begin{array}{c}\text { Insatisfecho } \\
\text { insatisfecho }\end{array}$ & 7 & $3.50 \%$ \\
\hline $\begin{array}{c}\text { Total } \\
\text { Muy }\end{array}$ & 200 & $19.50 \%$ \\
\hline
\end{tabular}

Tabla 1. Capacidad de reacción ante un imprevisto
En sentido general, los usuarios que acuden a recibir atención de parte de una secretaria ante un requerimiento, se encuentran satisfechos, específicamente porque en la mayoría de los casos, los secretarios atienden asuntos rutinarios que a fuerza de repetición saben el tipo de respuesta que deben dar al requerimiento, sin embargo, es importante destacar que se considera bajo el porcentaje $(21 \%)$ de usuarios que se encuentran totalmente satisfechos por la capacidad de reacción.

En la tabla 2 se puede evidenciar una coherencia con los resultados de la tabla 1 , en este caso el rango de valoraciones a las respuestas es semejante, demostrando que no se logra una atención de excelencia por parte de los secretarios, ya que es reducido el porcentaje que manifestó estar muy satisfecho por la actitud y cortesía de los secretarios que los atendieron, los resultados se presentan en la tabla 2 .

\begin{tabular}{|c|c|c|}
\hline Opciones & Frecuencia & Porcentaje \\
\hline Muy satisfecho & 45 & $22.5 \%$ \\
\hline Satisfecho & 97 & $48.5 \%$ \\
\hline Algo satisfecho & 20 & $10.0 \%$ \\
\hline Insatisfecho & 4 & $2.0 \%$ \\
\hline $\begin{array}{c}\text { Muy } \\
\text { insatisfecho }\end{array}$ & 34 & $17.0 \%$ \\
\hline \begin{tabular}{c} 
Total \\
\hline
\end{tabular} & $\mathbf{2 0 0}$ & $\mathbf{1 0 0 \%}$ \\
\hline
\end{tabular}

Tabla 2. Actitud y cortesía de la secretaria que lo atendió durante su visita

En cuanto a la percepción que tienen los usuarios respecto a la preparación y capacitación que tenían los secretarios que los atendió, de igual forma, en la tabla 3 se demuestra que se puede considerar como reducido el porcentaje de usuarios que está muy satisfecho, concentrándose el mayor porcentaje en aquellos que sencillamente están satisfechos por la preparación que pudieron observar de los secretarios, se observa en la tabla 3.

\begin{tabular}{|l|l|l|}
\hline Opciones & Frecuencia & Porcentaje \\
\hline $\begin{array}{l}\text { Muy } \\
\text { satisfecho }\end{array}$ & 39 & $19.5 \%$ \\
\hline
\end{tabular}




\begin{tabular}{|l|c|c|}
\hline Satisfecho & 98 & $49.0 \%$ \\
\hline $\begin{array}{l}\text { Algo } \\
\text { satisfecho }\end{array}$ & 28 & $14.0 \%$ \\
\hline Insatisfecho & 1 & $0.5 \%$ \\
\hline $\begin{array}{c}\text { Muy } \\
\text { insatisfecho }\end{array}$ & 34 & $17.0 \%$ \\
\hline Total & 200 & $100 \%$ \\
\hline
\end{tabular}

Tabla 3. Preparación y capacitación de la secretaria que lo atendió

En la tabla 4 muestra los resultados del ítem que consultaba a los usuarios sobre el nivel de satisfacción por el tiempo de espera para ser atendidos por los secretarios; en este sentido, se puede notar un ligero aumento en cuanto a la opción de muy satisfechos que alcanza el $24.5 \%$, sin embargo el mayor porcentaje se sigue situando en la opción de "satisfecho". Esta forma de marcar la opción de "satisfecho" como alternativa de respuesta, pone de manifiesto un poco la indiferencia de los usuarios frente a la atención que tuvieron, quizás por estar acostumbrados a la misma atención o tal vez porque se distrajo con otros asuntos que no prestó atención en los detalles de la atención recibida.

\begin{tabular}{|l|l|l|}
\hline Opciones & Frecuencia & Porcentaje \\
\hline Muy satisfecho & 49 & $24.5 \%$ \\
\hline Satisfecho & 98 & $49.0 \%$ \\
\hline Algo satisfecho & 18 & $9.0 \%$ \\
\hline Insatisfecho & 4 & $2.0 \%$ \\
\hline Muy insatisfecho & 31 & $15.5 \%$ \\
\hline Total & 200 & $100 \%$ \\
\hline
\end{tabular}

Tabla 4. Tiempos de espera para ser atendidos

De forma general, se sigue marcando la tendencia, el mismo rango en las respuestas a "muy satisfactorio" por las respuestas a los requerimientos y se acentúa el mayor porcentaje en la opción "satisfecho", confirmando lo dicho en el análisis de la tabla 4, es decir que los usuarios no observan al detalle la calidad de atención recibida o quizás les resulta indiferente el trato recibido, se observa en la tabla 5.

La reflexión en torno al empleo a la facilidad de diálogo con la secretaria, se muestra en las respuestas a la facilidad de diálogo, resultados en los cuales se mantiene la tendencia, confirmando una vez más lo expresado en los análisis anteriores, se observa en la tabla 6.

\begin{tabular}{|c|c|c|}
\hline Opciones & Frecuencia & Porcentaje \\
\hline Muy satisfecho & 44 & $22.0 \%$ \\
\hline Satisfecho & 69 & $34.5 \%$ \\
\hline Algo satisfecho & 37 & $18.5 \%$ \\
\hline Insatisfecho & 16 & $8.0 \%$ \\
\hline Muy insatisfecho & 34 & $17.0 \%$ \\
\hline Total & 200 & $100 \%$ \\
\hline
\end{tabular}

Tabla 5. Satisfacción por las respuestas a sus requerimientos

\begin{tabular}{|c|c|c|}
\hline Opciones & Frecuencia & Porcentaje \\
\hline Muy satisfecho & 45 & $22.5 \%$ \\
\hline Satisfecho & 68 & $34.0 \%$ \\
\hline Algo satisfecho & 39 & $19.5 \%$ \\
\hline Insatisfecho & 12 & $6.0 \%$ \\
\hline Muy insatisfecho & 36 & $18.0 \%$ \\
\hline Total & 200 & $100 \%$ \\
\hline
\end{tabular}

Tabla 6. Facilidad de diálogo con la secretaria

\section{Discusión}

Dentro del área profesional el perfil de la Secretaria Ejecutiva, ha evolucionado de manera importante en las últimas décadas, como consecuencia de las diversas demandas del mercado, de las empresas y de la sociedad, oriunda de factores como la globalización, la economía y la política. Anteriormente las exigencias eran menores y posteriormente se encontraban con labores sencillas. El perfil profesional de los secretarios ejecutivos se ha trasformado en base al continuo cambio de los equipos, funciones, requisitos y formación profesional, en el desarrollo de mayores responsabilidades orientadas a mayor especialización, se concuerda en esta investigación con (Cam, 2014). 
Se ha apreció en 100 secretarios (50\%) que el comportamiento no fue ético durante sus conversaciones con los jefes y usuarios, permitiéndose vislumbrar que aun cuando son competentes en su campo, la forma en que se comportan no les permite obtener su promoción en el momento debido.

Se coincide en esta investigación en el comportamiento negativo de los secretarios conduce a algo que no suponen críticamente tener. Por lo tanto, es necesario examinar el comportamiento, los personajes y la conducta que se espera de los secretarios en los últimos tiempos para determinar si existe una medida de mejora en sus comportamientos éticos (Ciulla, 2009).

En el mundo del trabajo actual, se espera que los secretarios o gerentes de oficina, según sea el caso, contribuyan significativamente a los objetivos de la organización al proporcionar la información necesaria y también promover la imagen de la organización en el mundo. Hay altas expectativas de los empleadores, colegas, clientes, visitantes y otros en el transporte de los deberes y roles de los secretarios / gerentes de oficina (Ciulla, Price, \& Murphy 2005).

El 100\% de las empresas objeto de la investigación, se constató que esperan que los profesionales de la secretaría se comuniquen claramente, tengan buenas características personales, especialmente en términos de apariencia, buenas interacciones sociales, buena disposición hacia las personas. Elemento que se contrastó con los aportes de Dasborough \& Harvey (2016) quienes declararon que: “... buena conducta personal en términos de manejar a las personas con tácticas y respeto, tener una buena relación interpersonal, comportarse de una manera que refleje altos estándares morales de probidad personal, manteniendo los códigos de conducta y comportamiento como se espera de la profesión, así como la competencia técnica" (Dasborough \& Harvey, 2016).

Se apreció además que en el 50\% de las empresas, se demanda la existencia del perfil de los secretarios ejecutivo, acorde a las competencias profesionales en función del mejoramiento del desempeño.

La ética de una secretaria son principios morales relacionados con el trabajo que uno está obligado. Estos requisitos se toman automáticamente en cuenta cuando uno acepta cualquier puesto como secretario $\mathrm{y}$, por lo tanto, se espera que los cumpla en todo momento, al igual que el Sacerdote católico mantiene el secreto del sacramento de la reconciliación (Eisenbeiss \& van Knippenberg, 2015).

En el abordaje teórico y a partir del criterio de los 200 secretarios (100\%), se logró develar los valores éticos de los secretarios, por consenso:

- Confidencialidad, que se aprecia en mantener siempre la información privada y confidencial sobre la empresa para la que trabaja y sus clientes. Nunca deben repetir información confidencial, incluso si están en una discusión donde todos se preguntan qué está pasando y el trabajador lo sabe. Deben siempre ser confiable (Elfenbein, 2007).

- Lealtad: Deben ser siempre leales a sus jefes y compañía. Nunca deben vender a cualquier costo. Sin embargo, una vez dicho esto, el jefe también tiene que demostrar que es digno de su lealtad.

- Honestidad: No deben tomar el crédito por algo que no se hizo y no deben permitir que otra persona disfrute del crédito por algo que él / ella no hizo. Siempre deben ser sinceros.

- Fiable: Deben demostrar que son confiables, además demostrar la puntualidad para el trabajo y las reuniones en todo momento, siempre deben llevar la documentación pertinente a las reuniones, Tienen que asegurar que cada tarea que se le asigne se complete a tiempo y lo mejor que pueda.

- Responsabilidad: Deben demostrar que son responsables estableciendo prioridades y realizando tareas de manera oportuna. Siempre deben cumplir con los plazos cuando se les da. Si delega, siempre verifique que el proyecto esté en buen camino. No olvidar simplemente porque alguien más lo está haciendo. Deben mantener siempre actualizado el trabajo sin supervisión.

Es fundamental reconocer que el trabajo profesional de los secretarios camine de la mano con la aplicación de la ética profesional y valores que debe poseer, se implica en la reservación secreta de la información, por el trabajo que realiza, ya que ella tiene acceso a la mayoría de las documentaciones, que contienen en muchos casos comunicación reservada (Silcock \& Duncan, 2001).

En la actualidad ecuatoriana, la secretaria participa en el proceso administrativo intrínsecamente con las 
instituciones, y representa un factor decisivo en el desempeño laboral, deben situar y guiar en la realización de trámites, solicitudes, dar asesoramientos, planificar diversas actividades de carácter administrativo, entre muchas otras. Por estas razones para tener éxito en los diversos campos laborales, el secretario debe contar con, hábitos de estudio, actitudes, motivación habilidad verbal, redacción, comunicación, organización pero sobre todo efectividad para lograr cada una de sus funciones en el ámbito organizacional e institucional.

Muchas veces se relaciona al apoyo y la vigilancia de los procesos en el que debe ser una persona en la que se tenga plena confianza, excelente redacción y ortografía, facilidad de expresión verbal y escrita, persona proactiva, y organizada, facilidad para interactuar en grupos, dominio de programas ofimáticos, brindar apoyo a todos los departamentos, desempeñarse eficientemente en cualquier área administrativa, conocimientos en el área de logística, atención al cliente, aptitudes para la organización, buenas relaciones interpersonales, dinámica y entusiasta, habilidades para el planeamiento, innovación, motivación, liderazgo y toma de decisiones, capacidad de trabajar en equipo y bajo presión. Por lo mencionado anteriormente las habilidades forman parte principal en el desarrollo de las actividades secretariales, al mismo tiempo ser eficaz y eficiente para así lograr y cumplir con cada una de sus funciones.

En sentido general, se necesita que los secretarios posean una solidez actitudinal: la empatía, comunicación y el esfuerzo por despejar el camino o simplificar las tareas son cualidades importantes, asumir diferentes responsabilidades y funciones así como contar con la facilidad para coordinarla. Siendo así la secretaria una de las colaboradoras más cercanas a los directivos, en la que deberá poseer los conocimientos y cualidades para saber producir el área en la que se desenvuelva.

Es por ello que resulta fundamental desarrollar habilidades no solo actitudinales sino también profesionales. La capacitación y el entrenamiento continuo son primordiales, además de contar con una formación completa en temas de cultura general y del ámbito político y económico esto dependerá de la entidad resulta indispensable sus competencias técnicas y sus conocimientos específicos de acuerdo al área al que deba asistir.

De igual manera es imprescindible el uso de herramientas ofimáticas, pues son competencias en las que se enfrenta, estar actualizado en los diversos campos que ofrece la tecnología como también conocer otras especialidades que sean relacionados en el desempeño de cada una de las funciones. Los secretarios deben conocer todos los programas ofimáticos, conocer otras materias como economía, marketing o derecho; además de tener una buena preparación administrativa, pero existen numerosos puestos de secretariado que harán que ciertas disciplinas sean más prioritarias que otras (de Klerk, 2016).

\section{Conclusión}

La dimensión ética de los secretarios, permite identificar a grandes rasgos el perfil éticoprofesional que proponen para delinear las características, los principios y los valores que requieren estas profesionales para desarrollar un ejercicio profesional ético. Sin embargo, es común que los códigos éticos se interpreten como un conjunto de normas formales que acepta y reconoce el profesional más por conveniencia que por convicción. Por ello, para que los futuros secretarios puedan asumir la dimensión ética de la profesión, es necesario que las instituciones de educación superior abran espacios o fortalezcan la formación ética en sus programas para que durante la formación en la educación superior se vayan incorporando y asimilando aspectos éticos de la profesión como una característica que enriquece la condición de profesionales.

\section{Bibliografía}

Ahrne, G., \& Brunsson, N. (2010). Organization outside organizations: the significance of partial organization. Organization.

Alford, C. (2001). Whistleblowers: broken lives and organizational power. . NY: Cornell University Press.

Allen, M. (2002). The business of genocide: The SS, slave labor, and the concentration camps. . NC: University of North Carolina Press.

Arce, J., \& Macías, J. (2015). El perfil profesional de las secretarias ejecutivas y la demanda en el mercado laboral de las instituciones públicas de la ciudad de Portoviejo (Tesis de pregrado). UNIVERSIDAD TÈCNICA DE MANABİ.

Artico, J. (2013). O Secretário Executivo Com Perfil De Relações Públicas. Revista de Gestão e Secretariado, 4 (1), 126-138. . 
Bermúdez, M., \& Chila, D. (2015). Las competencias comunicativas de las secretarias ejecutivas y su desempeño laboral en las entidades públicas de la ciudad de Portoviejo, 2014. Portoviejo, Manabi, Ecuador.

Brown, A., Gabriel, Y., \& Gherardi, S. (2009). Storytelling and change: An unfolding story. . Organization, 16(3), 323-333.

Cam, P. (2014). Philosophy for children, values education and the inquiring society. Educational Philosophy and Theory, , 46(11), 1203-1211.

Ciulla, J. (2009). Leadership and the ethics of care. . Journal of Business Ethics, 88(1), 3-4. .

Dasborough, M., \& Harvey, P. (2016). Schadenfreude: The (not so) Secret Joy of Another's Misfortune. . Journal of Business Ethics.

Eisenbeiss, S., \& van Knippenberg, D. (2015). On ethical leadership impact: The role of follower mindfulness and moral emotions. . Journal of Organizational Behavior 36(2), , 182-195.

Gabriel, Y. (2015). The caring leader-What followers expect of their leaders and why? Leadership, 316-334.

Ryan, K. (2015). A brief history of moral education, the return of character education, current approaches to moral education. Moral education. 